\title{
Study on the efficacy of neoadjuvant chemotherapy on limb salvage surgery for osteosarcoma.
}

\author{
Jun Zhao* \\ Changzhi Medical College, Shanxi, PR China
}

\begin{abstract}
In this study, 32 osteosarcoma patients admitted in the department of orthopedics from 2008 to 2016 were selected as subjects. Percutaneous intra-arterial chemotherapy was performed on 17 patients on the day before admission, and then the general chemotherapy was carried out on next day. After limb salvage surgery, the previous chemotherapy was repeated for 4 times. Before limb salvage surgery, the remaining 15 patients were treated with intravenous systemic chemotherapy. Fourteen days later, amputation was performed. Both the treatment strategies were compared and it was concluded that patients can adapt better to the method of limb salvage treatment after arterial and venous chemotherapy, thus the application of which is more promising.
\end{abstract}

Keywords: Osteosarcoma, Neoadjuvant chemotherapy, Amputation, Limb salvage, Surgical treatment.

Accepted on May 23, 2017

\section{Introduction}

In the cycle of human growth and development, 15-25 age groups are more susceptible to osteosarcoma/osteogenic sarcoma and related symptoms. Neo-adjuvant chemotherapy is often combined with the limb salvage surgeries and this has found to improve the survival of the patients of younger age group [1]. The rate of incidence of osteosarcoma is higher in males than females. In general, among sick patients, out of every three people two are male and one is female [2]. From a statistical point of view, although, the disease is mainly seen in children and adolescents, it can actually occur in every age group [3]. For the patients in the other group, the intravenous amputation is used. The effects of two kinds of chemotherapy on limb salvage surgery for osteosarcoma are compared and analyzed.

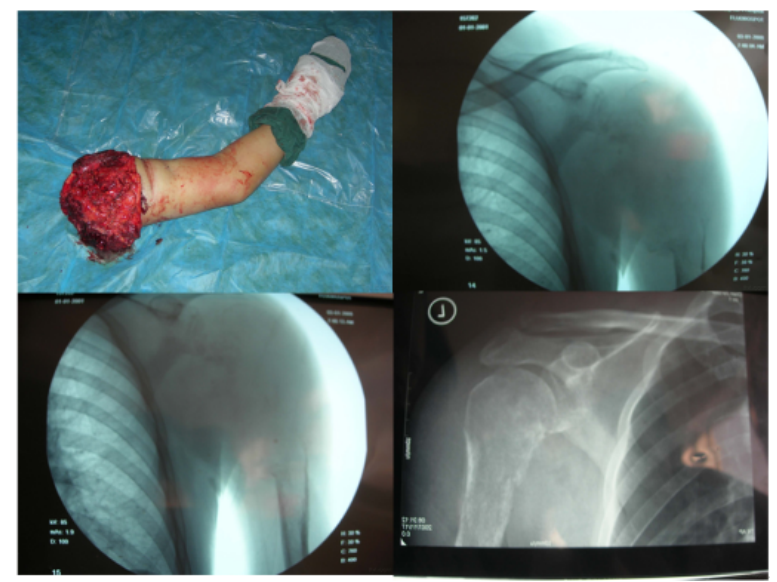

Figure 1. The imaging results of left bone osteoma.

\section{State of the Art}

After chemotherapy, the patient's pain will be gradually reduced, and the sleep quality can be improved significantly. The results of clinical medicine show that the volume reduction of osteosarcoma is a good symptom after treatment. The volume changes of osteosarcoma after operation can be determined by measuring the circumference and diameter of the tissue [4]. The conventional X-ray imaging can describe the reduction of soft tissue volume in the vicinity of osteosarcoma, and can also be used to characterize the edema in vivo. The increase of periosteum and ossification show that the effect of chemotherapy is obvious, as shown in Figure 1.

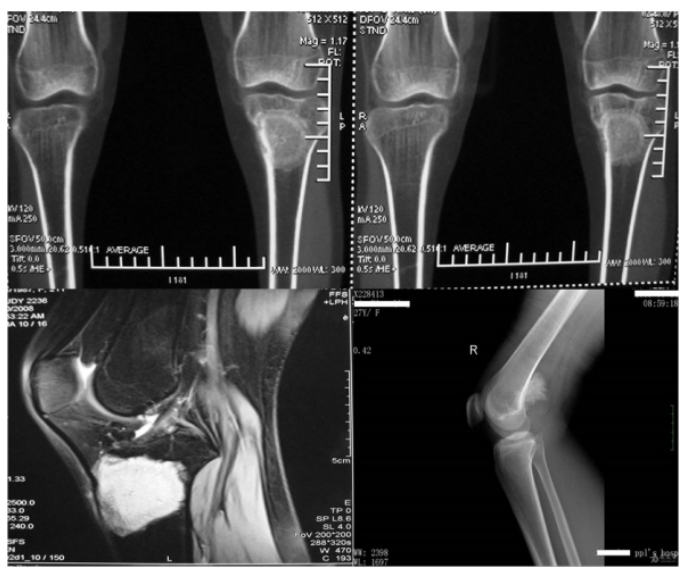

Figure 2. Results of osteosarcoma MRI.

To evaluate the efficacy of chemotherapy, observational and statistical analysis of osteosarcoma tumor volume change is measured mainly through the MRI imaging technology (Figure 
2). Some studies have suggested that $T 2$ signal can be associated with the recovery of osteosarcoma, which can be used to determine the changes of osteosarcoma with the increasing number of tissue signals [5].

\section{Methodology}

The selected age group of the patients ranges from 15 to 65 years, of which, there are 18 males, and the number of females is about 14. Table 1 shows the basic information of patients.

Table 1. Basic information of the research object.

\begin{tabular}{|c|c|c|}
\hline \multirow{2}{*}{ Age } & Average age & $30 \pm 5$ \\
\hline & $\operatorname{Max} / \min$ & $65 / 15$ \\
\hline \multirow{2}{*}{ Gender } & Male/female & $18 / 14$ \\
\hline & Male to female ratio & $1.28: 1$ \\
\hline \multirow{4}{*}{ Clinical manifestation } & Femur injury: 12 & \\
\hline & Tibia injury: 14 & \\
\hline & $\begin{array}{l}\text { There were } 23 \text { tumors with a } \\
\text { diameter less than } 10 \mathrm{~cm}\end{array}$ & \\
\hline & $\begin{array}{l}\text { There are } 9 \text { sites with a } \\
\text { diameter greater than } 10 \mathrm{~cm}\end{array}$ & \\
\hline \multirow{2}{*}{ Surgical detection } & $\alpha A$ & $\alpha B$ \\
\hline & 11 & 21 \\
\hline
\end{tabular}

The results show the clinical manifestations of bone condition. There are 12 locations at the lower end of the femur. At the lower end of the tibia, there are 14. And there are 3 locations on the upper end of the femur.

Multi-agent chemotherapy combined with limb salvage surgery approach has been adopted for the amputation [6]. DDP is the drug mainly used for intra-arterial infusion chemotherapy, $\mathrm{ADM}$ is used for intravenous chemotherapy, and the combination of DDP and ADM is used for the amputation of chemotherapy. The contents of DDP and ADM in two groups of $A$ and $B$ are the same. The content of DDP is $120 \mathrm{mg} / \mathrm{m}^{2}$, and the content of ADM is $70 \mathrm{mg} / \mathrm{m}^{2}$, as shown in Table 2 .

Table 2. Treatment model design of Group A and B.

\begin{tabular}{llllll}
\hline Project & $\begin{array}{l}\text { Numbe } \\
\mathbf{r}\end{array}$ & Drug type & $\begin{array}{l}\text { Drug } \\
\left(\mathbf{m g} / \mathbf{m}^{2}\right)\end{array}$ & content & $\begin{array}{l}\text { Medication } \\
(\mathbf{h})\end{array}$ \\
\hline Group A & 17 & ADM+DDP & ADM/70, DDP/120 & 24 \\
\hline Group B & 15 & ADM & ADM/70 & 96 \\
\hline
\end{tabular}

\section{Result Analysis and Discussion}

The patients in the A group and the B group are treated with two categories of preoperative chemotherapy regimens. In the volume test, all the osteosarcoma in vivo is decreased. With the limb salvage treatment, the magnitude of osteosarcoma volume reduction is the largest, and the patient's pain is shorter (Figure 3). There is no significant difference between the limb salvage surgery and amputation surgery in the long-term treatment of osteosarcoma. Since the results of limb salvage surgery provides a better improvement in terms of function, and pain relief, the chances of patients choosing it will be more. The study results thus provide a more promising treatment model for osteosarcoma patients.

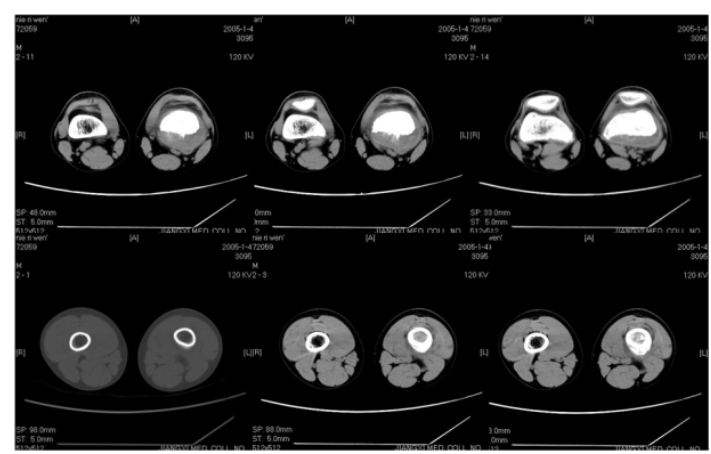

Figure 3. Effects of two preoperative chemotherapy.

In this study, limb salvage surgery is adopted. The surgical methods of osteosarcoma segment resection and artificial limb replacement are selected. Before surgery, the prosthetic limb is prepared for the patient. During the operation, the osteosarcoma is removed to the maximum extent. Figure 4 is the survival probability curve of limb salvage surgery, and the Figure 5 is the survival curve of patients after amputation.

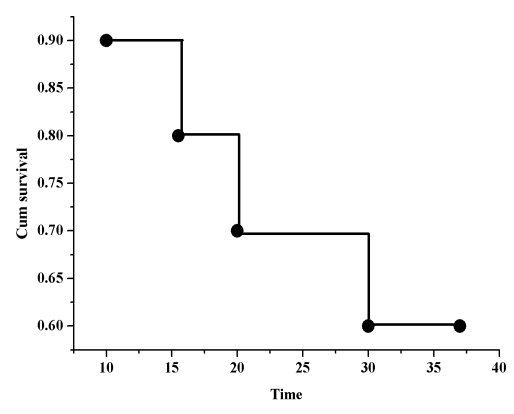

Figure 4. Survival probability curves of limb salvage surgery patients.

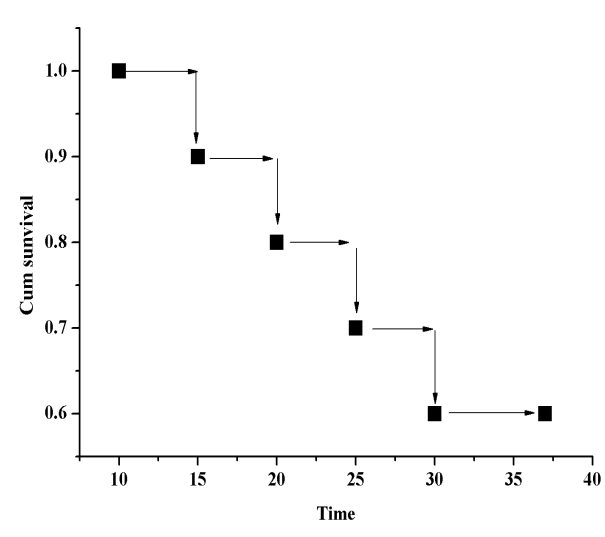

Figure 5. Survival curve of patients after amputation. 


\section{Conclusions}

Osteosarcoma is one of the challenging diseases in terms of disease management and recovery. This suggested mode can be a cost-effective method of treatment. The preliminary findings of this study promise a better improvement in terms of pain management and survival. Agarwal et al. also had a similar study report where they have showed $100 \%$ cell death and survival by using chemotherapy combined with limb salvage surgery [7]. The neoadjuvant chemotherapy also shows improvement in higher age groups of 41-60 years in a recent report by Bacci et al. [8].

In this study, 32 osteosarcoma patients in the department of orthopedics in a hospital from 2008 to 2016 were selected as the subjects. And the effect of adjuvant chemotherapy in limb salvage surgery for osteosarcoma was analyzed. The subjects were divided into group A and group B. However, there is no significant difference in local recurrence rate between A group and B group after surgery. The reason for the better comprehensive effect of limb salvage group is that the treatment of the limb salvage group can preserve the basic function of the affected limb, which can furthest improve the quality of life of patients. In addition, the effect of chemotherapy can achieve the purpose of killing tumor cells, so that the toxic reaction of patients can be reduced [9].

\section{References}

1. Longhi A, Errani C, De Paolis M, Mercuri M, Bacci G. Primary bone osteosarcoma in the pediatric age: state of the art. Cancer Treat Rev 2006; 32: 423-436.

2. Zheng K, Yu X, Chang Z. Effect of pathological fracture on limb salvage surgery with preservation of the epiphysis in children with osteosarcoma of the distal femur: Two case reports. Mol Clin Oncol 2016; 4: 523.
3. Kamal AF, Widyawarman H, Husodo K. Clinical Outcome and Survival of Osteosarcoma Patients in Cipto Mangunkusumo Hospital: Limb Salvage Surgery versus Amputation. Acta Medica Indonesiana 2016; 48: 175.

4. Shin KH, Moon SH, Suh JS, Yang WI. Tumor volume change as a predictor of chemotherapeutic response in osteosarcoma. Clin Orthopaedics Related Rese 2000; 376: 200-208.

5. Bydder GM, Young IR. MR imaging: clinical use of the inversion recovery sequence. Journal of computer assisted tomography. 1985; 9: 659-675.

6. Tiwari A, Jain S, Mehta S, Kumar R, Kapoor G, Kumar K. Limb salvage surgery for osteosarcoma: Early results in Indian patients. Indian J Orthopaedics 2014; 48: 266-272.

7. Agarwal M, Anchan C, Shah M, Puri A, Pai S. Limb salvage surgery for osteosarcoma: effective low-cost treatment. Clin Orthop Relat Res 2007; 459: 82-91.

8. Bacci G, Ferrari S, Mercuri M. Neoadjuvant chemotherapy for osteosarcoma of the extremities in patients aged 41-60 years. Acta Orthopedica 2007.

9. Xing $\mathrm{Wu}$, Zheng-dong Cai, Zheng-rong Chen, Zhen-jun Yao, Guang-jian Zhang. A Preliminary Evaluation of Limb Salvage Surgery for Osteosarcoma around Knee Joint. Plos one 2012.

\section{*Correspondence to}

Jun Zhao

Changzhi Medical College

PR China 